\title{
THEORETICAL PREDICTION OF VIBRATIONAL AND ROTATIONAL SPECTRA. FORMYL CYANIDE, HCOCN, AND THIOFORMYL CYANIDE, HCSCN
}

\author{
Attila G. CSÁSZÁR \\ Laboratory of Theoretical Chemistry, Eötvös Loránd University, Múzeum krt. 6-8, 1088 Budapest, Hungary
}

Received 28 July 1989; in final form 8 August 1989

\begin{abstract}
Optimized geometries and complete, scaled quadratic force fields of HCOCN and HCSCN have been determined at different theoretical levels (HF/4-21, HF/6-31G $\mathrm{G}^{* *}$; geometries also at $\mathrm{MP}^{2} / 6-31 \mathrm{G}^{* *}$ and $\left.\mathrm{MP}^{2} / 6-311 \mathrm{G}^{* *}\right)$. Frequencies calculated from the force fields confirm, with one exception, the assignment of the vibrational spectrum of HCOCN. The vibrational fundamentals calculated for $\mathrm{HCSCN}$ (accurate within about $50 \mathrm{~cm}^{-1}$ ) could direct a spectroscopy study aimed at determining them. Calculated rotational and quartic centrifugal distortion (QCD) constants are in good agreement with the experimental data for both molecules, but QCD constants only after scaling of the force fields.
\end{abstract}

\section{Introduction}

The first spectroscopic detection of the both astrophysically and chemically very interesting but rather unstable molecule of formyl cyanide, HCOCN, was performed by Judge et al. [1]. The near-UV absorption spectrum has been recorded [1], and partial analysis of the vibrational bands and the rotational fine structure was presented: besides the lowlying seven vibrational fundamentals of the lowest electronically excited state of formyl cyanide, two low-lying ground state vibrational fundamentals have been obtained ( $\nu_{7}$ and $\nu_{9}$, belonging to the CCN bending modes). Four of the seven in-plane fundamentals $\left(\nu_{2}, \nu_{3}, \nu_{5}\right.$ and $\left.\nu_{7}\right)$ have been observed from the gas-phase infrared spectrum by Clouthier and Moule [2]. The $a$-type millimeter-wave (MMW) spectrum, measured by Bogey et al. [3], provided the rotational, the quartic centrifugal distortion and two of the seven sextic constants. (In this study one of the quartic centrifugal distortion constants, $\Delta_{K}$, was fixed at its propynal value because only $a$-type transitions were measured, and $\Delta_{K}$ was found to be highly correlated with the $A$ constant.) Measurement of the $b$-type spectrum provided refined values for all these molecular constants [4].

The only spectroscopic work published on monomeric, gas-phase thioformyl cyanide, $\mathrm{HCSCN}$, is that of Bogey et al. [5], who investigated the MMW spectrum, measured lines belonging to the $a$ - and $b$ type spectra and derived accurate molecular constants (besides the full quartic and sextic terms, they also obtained an octic and dectic centrifugal distortion constant ). Their attempt to observe HCSCN by low temperature IR spectroscopy was, however, unsuccesful.

As to the theoretical calculations on these simple compounds, Goddard [6] determined the geometry and the vibrational frequencies of $\mathrm{HCOCN}$ at the $\mathrm{ab}$ initio SCF level using the $3-21 \mathrm{G}$ and $6-31 \mathrm{G}^{*}$ basis sets, and the geometry of HCOCN also at the MP3/ 6-31G* level. In the calculation of the rotational constants of HCOCN Goddard used some empirical scaling, and a uniform scaling was applied in the calculation of its vibrational fundamentals. To our best knowledge similar theoretical calculations on HCSCN are not available.

Force fields for HCOCN and HCSCN have not been reported so far, although knowledge of the harmonic force field provides the best way to check the assignment of the vibrational spectrum and the molecular constants deduced from the MMW spectrum. Force fields are also essential in understanding the fine details of the electronic structure of a molecule. Therefore we felt it is necessary to obtain reliable quadratic force fields for HCOCN and HCSCN. 
(During the course of the calculation of the frequencies for $\mathrm{HCOCN}$, Goddard [6] actually did calculate a force field for this molecule. However, he did not report it in his paper, furthermore he did not attempt to systematically correct the force field, without which the results have only limited value, as is again demonstrated in this paper.)

It has been proved several times that molecular orbital theory can predict highly accurate geometries (the rotational constants are correct mostly well within $1 \%$ ) and that quantum mechanical force fields (especially after some empirical adjustment [7]) give very good estimates of the vibrational fundamentals of the ground states of molecules [7-11] and that they successfully reproduce the quartic centrifugal distortion constants [12-14]. Among mediumsized calculations (Hartree-Fock SCF) the most careful and thorough harmonic force field investigations involve the 4-21 Gaussian basis set [11]. For the sake of consistency and for an easier judgment of the theoretical results it is still probably worthwhile choosing this theoretical level (may be denoted as HF/4-21) for molecules with regular bonding. Thus results of such a calculation are reported here for HCOCN. On the other hand, feasibility of much larger calculations (more sophisticated theoretical methods with more complete basis sets) is steadily improving. Therefore we also made calculations with a larger basis set $\left(6-31 G^{* *}[15]\right)$. The following is an account of theoretical calculations of the geometries and SQM force fields of formyl cyanide and thioformyl cyanide, which data were used to calculate their vibrational and rotational spectra in the usual fashion [16].

\section{Computational details}

Electronic wave function calculations were made at two levels of theory: (a) the self-consistent-field (SCF) Hartree-Fock (HF) method; (b) the MollerPlesset second-order (MP2) perturbational method. Three basis sets were used in this study: (a) Pulay's 4-21 basis [11]; (b) Pople's 6-31G** basis [15]; (c) the $6-311 \mathrm{G}^{* *}$ basis [17]. The actual calculations were carried out using either the MONSTERGAUSS [18] or the GAUSSIAN 86 [19] program systems. The HF level calculations were made with the two smaller basis sets, while the MP2 geometry optimizations were performed with the two larger basis sets. The geometries were optimized by analytic gradient methods; the HF/6-31G** force field calculations made use of the analytic second derivatives package of GAUSSIAN 86.

The internal coordinate system used for both HCOCN and HCSCN was selected in accordance with the recommendations of ref. [11], and is listed in table 1.

In obtaining the geometry and the scaled quantum mechanical (SQM) HF/4-21 force field for HCOCN a well-documented, standard route advocated by $\mathrm{Pu}$ lay et al., described in detail in refs. [7,11], is followed. The reference geometry is obtained by empirical correction of the theoretical equilibrium geometry, determined by complete optimization, using the following offset values $[7,20]:+0.016 \AA$ for the $\mathrm{CH}$ and $\mathrm{CN},-0.001 \AA$ for the $\mathrm{CC}$ and -0.005 $\AA$ for the $\mathrm{CO}$ bond (for the final results see table 2). Scaling of the force constants was also done as described previously [7]. In this scheme, applied throughout this study, scale factors represent simultaneous empirical corrections for both the deficiency of the theoretical model and for anharmonicity. The scale factors were, when appropriate, taken from the standard set recommended for such calculations [7]. The scale factors for the $\mathrm{C}=\mathrm{N}$ stretching and $\mathrm{CCN}$ bending motions were taken from similar HF/4-21 calculations on $\mathrm{HCN}$ [20]. These standard scale factors are listed in table 1, the SQM force field in table 3.

No correction of the fully optimized theoretical geometries was attempted at any other theoretical level. Thus the theoretical geometries presented in table 2 refer to geometries used in this study for the evaluation of the quadratic force constants.

As was pointed out several times (for some fascinating new results see ref. [21], a study interesting also in this respect), even at higher theoretical levels adjustment of the computed force constants is necessary to obtain chemically accurate (with errors less than $1-1.5 \%$ ) values for the measured fundamentals. Unfortunately no scale factors within the scaling scheme used in this study are available for the HF/6-31G** level from the literature. Thus scale factors were obtained by a least-squares refinement procedure [7], in which the calculated HF/6-31G** 
Table 1

Internal coordinates and scale factors ${ }^{2)}$

\begin{tabular}{|c|c|c|c|c|}
\hline \multirow[t]{2}{*}{ No. } & \multirow[t]{2}{*}{ Internal coordinate } & \multirow[t]{2}{*}{ Description } & \multicolumn{2}{|c|}{ Scale factor ${ }^{b)}$} \\
\hline & & & $\mathrm{HF} / 4-21$ & $\mathrm{HF} / 6-31 \mathrm{G}^{* *}$ \\
\hline \multicolumn{5}{|c|}{ in-plane } \\
\hline 1 & $r_{\mathrm{CC}}$ & CC stretch $(\mathrm{CC})$ & 0.920 & 0.85 \\
\hline 2 & $r_{\mathrm{CX}}$ & $\mathrm{CO} / \mathrm{CS}$ stretch $(\mathrm{CO} ; \mathrm{CS})$ & 0.826 & 0.71 \\
\hline 3 & $r_{\mathrm{CH}}$ & $\mathrm{CH}$ stretch $(\mathrm{CH})$ & 0.866 & 0.85 \\
\hline 4 & $r_{\mathrm{CN}}$ & $\mathrm{CN}$ stretch $(\mathrm{CN})$ & 0.850 & 0.71 \\
\hline 5 & $\alpha_{\mathrm{CCN}}$ & $\mathrm{CCN}$ bend $(\beta \mathrm{CCN})$ & 0.650 & 0.71 \\
\hline 6 & $\alpha_{\mathrm{CCX}}-\alpha_{\mathrm{HCX}}-\alpha_{\mathrm{HCC}}$ & $\mathrm{CCO} / \mathrm{CCS}$ bend $(\beta \mathrm{CCO} ; \beta \mathrm{CCS})$ & 0.836 & 0.82 \\
\hline 7 & $\alpha_{\mathrm{HCX}}-\alpha_{\mathrm{HCC}}$ & $\mathrm{CH}$ rock $(\beta \mathrm{CH})$ & 0.836 & 0.82 \\
\hline \multicolumn{5}{|c|}{ out-of-plane } \\
\hline 8 & $\mathrm{H}$ out of CCX plane & $\mathrm{CH}$ wag $(\gamma \mathrm{CH})$ & 0.730 & 0.82 \\
\hline 9 & $\gamma \mathrm{CCN}$ & $\mathrm{CCN}$ bend $(\gamma \mathrm{CCN})$ & 0.650 & 0.71 \\
\hline
\end{tabular}

a) $\mathrm{X}=\mathrm{O}$ for $\mathrm{HCOCN}$ and $\mathrm{X}=\mathrm{S}$ for $\mathrm{HCSCN}$. $\gamma_{\mathrm{CCN}}$ is the angle of the $\mathrm{NC}$ bond with the $\mathrm{C}_{1} \mathrm{C}_{2} \mathrm{O}_{3}$ plane. The $\mathrm{CH}$ wag is positive if the $\mathrm{H}$ atom moves toward the positive $Z$ direction.

b) The HF/4-21 scale factors were taken from refs. [7,20]; for the HF/6-31G** scale factors see text.

Table 2

Ab initio structural results for formyl cyanide and thioformyl cyanide ${ }^{\text {) }}$

\begin{tabular}{|c|c|c|c|c|c|c|c|c|}
\hline \multirow[t]{3}{*}{ Parameter } & \multicolumn{5}{|c|}{ Formyl cyanide } & \multicolumn{3}{|c|}{ Thioformyl cyanide } \\
\hline & \multicolumn{2}{|c|}{ HF-SCF } & \multicolumn{2}{|l|}{ MP2 } & \multirow{2}{*}{$\begin{array}{l}\text { MP3 }{ }^{\mathrm{c}} \\
6-31 \mathrm{G}^{*}\end{array}$} & \multirow{2}{*}{$\begin{array}{l}\text { HF-SCF } \\
6-31 G^{* *}\end{array}$} & \multicolumn{2}{|l|}{ MP2 } \\
\hline & $4-21^{b)}$ & $6-31 G^{* *}$ & $6-31 G^{* *}$ & $6-311 G^{* *}$ & & & $6-31 \mathrm{G}^{* *}$ & $6-311 G^{* *}$ \\
\hline$r\left(\mathrm{C}_{1}-\mathrm{C}_{2}\right)$ & 1.457 & 1.478 & 1.469 & 1.473 & 1.476 & 1.443 & 1.434 & 1.434 \\
\hline$r\left(\mathrm{C}_{1}-\mathrm{X}_{4}\right)$ & 1.200 & 1.180 & 1.220 & 1.210 & 1.207 & 1.597 & 1.624 & 1.622 \\
\hline$r\left(\mathrm{C}_{1}-\mathrm{H}_{5}\right)$ & 1.094 & 1.086 & 1.096 & 1.100 & 1.098 & 1.078 & 1.086 & 1.091 \\
\hline$r\left(\mathrm{C}_{2}-\mathrm{N}_{3}\right)$ & 1.153 & 1.134 & 1.182 & 1.175 & 1.160 & 1.136 & 1.184 & 1.178 \\
\hline$\angle \mathrm{NCC}$ & 179.8 & 178.0 & 179.3 & 179.3 & 178.3 & 178.6 & 179.2 & 179.4 \\
\hline$\angle \mathrm{XCC}$ & 123.0 & 121.9 & 121.9 & 122.1 & 121.6 & 124.0 & 123.5 & 123.4 \\
\hline$\angle \mathrm{HCC}$ & 114.1 & 114.4 & 114.9 & 114.5 & 115.2 & 114.0 & 115.1 & 115.1 \\
\hline dip. mom. & 2.64 & 2.77 & 2.82 & 2.80 & - & 2.71 & 2.73 & 2.72 \\
\hline
\end{tabular}

a) Distances ( $r$ ) in $\AA$, angles $(\angle)$ in deg, dipole moments in $D$. Numbering of atoms: $\mathrm{C}_{1} \mathrm{H}_{5} \mathrm{X}_{4}-\mathrm{C}_{2} \mathrm{~N}_{3}$, where $\mathrm{X}=\mathrm{O}$ for $\mathrm{HCOCN}$ and $\mathrm{X}=\mathrm{S}$ for HCSCN.

b) Corrected theoretical geometry (see text). ${ }^{\text {c) }}$ Ref. [6].

force field of HCOCN was adjusted to give frequencies in close agreement with the measured ones. The obtained scale factors, presented in table 1 , were used to correct the HF/6-31G** force field of HCSCN. The final SQM force fields of $\mathrm{HCOCN}$ and HCSCN, obtained with these scale factors, are presented in table 3 .

The measured and the calculated vibrational frequencies of HCOCN and HCSCN are presented in table 4. As scaling of the calculated vibrational frequencies using a unique scale factor is a widely applied procedure, results of this oversimplified procedure are also presented in this table for purpose of comparison with the more sophisticated results obtained by the scaling of the force field. The unique scale factor was chosen to be 0.89 for the HF/6$31 G^{* *}$ frequencies.

Calculation of the rotational and quartic centrif- 
Table 3

Quadratic SQM force fields of formyl cyanide and thioformyl cyanide ${ }^{\text {a) }}$

\begin{tabular}{|c|c|c|c|}
\hline \multirow[t]{2}{*}{$F_{i j}$} & \multicolumn{2}{|c|}{ Formyl cyanide } & \multirow{2}{*}{$\begin{array}{l}\text { Thioformyl cyanide } \\
\text { HF/6-31G** }\end{array}$} \\
\hline & $\mathrm{HF} / 4-21$ & $\mathrm{HF} / 6-31 \mathrm{G}^{* * *}$ & \\
\hline
\end{tabular}

\begin{tabular}{|c|c|c|c|}
\hline \multicolumn{4}{|c|}{ in-plane } \\
\hline 1,1 & 4.740 & 4.387 & 5.099 \\
\hline 2,1 & 0.987 & 0.962 & 0.419 \\
\hline 2,2 & 12.430 & 12.259 & 5.752 \\
\hline 3,1 & 0.141 & 0.146 & 0.110 \\
\hline 3,2 & 0.317 & 0.379 & 0.108 \\
\hline 3,3 & 4.607 & 4.942 & 5.178 \\
\hline 4,1 & -0.177 & -0.018 & 0.206 \\
\hline 4,2 & -0.051 & 0.008 & -0.076 \\
\hline 4,3 & 0.000 & 0.007 & -0.010 \\
\hline 4,4 & 17.884 & 17.276 & 17.023 \\
\hline 5,1 & 0.007 & 0.017 & 0.023 \\
\hline 5,2 & -0.022 & -0.039 & -0.025 \\
\hline 5,3 & 0.002 & 0.004 & 0.006 \\
\hline 5,4 & 0.007 & -0.012 & -0.004 \\
\hline 5,5 & 0.271 & 0.260 & 0.315 \\
\hline 6,1 & 0.197 & 0.141 & 0.096 \\
\hline 6,2 & 0.226 & 0.204 & 0.145 \\
\hline 6,3 & -0.128 & -0.092 & -0.070 \\
\hline 6,4 & -0.014 & 0.005 & 0.012 \\
\hline 6,5 & -0.128 & -0.111 & -0.072 \\
\hline 6,6 & 0.837 & 0.830 & 0.647 \\
\hline 7,1 & -0.262 & -0.224 & -0.198 \\
\hline 7,2 & 0.325 & 0.281 & 0.177 \\
\hline 7,3 & -0.034 & -0.026 & -0.063 \\
\hline 7,4 & -0.032 & -0.033 & -0.026 \\
\hline 7,5 & -0.044 & -0.044 & -0.044 \\
\hline 7,6 & -0.057 & -0.066 & -0.002 \\
\hline 7,7 & 0.628 & 0.600 & 0.522 \\
\hline \multicolumn{4}{|c|}{ out-of-plane } \\
\hline 8,8 & 0.348 & 0.393 & 0.295 \\
\hline 9,8 & -0.042 & -0.039 & -0.025 \\
\hline 9,9 & 0.288 & 0.227 & 0.281 \\
\hline
\end{tabular}

a) Units are consistent with energy measured in aJ, stretching coordinates in $\AA$ and bending coordinates in rad. The values listed are $a b$ initio values scaled with scale factors of table 1 . See table 1 for the definition of internal conrdinates.

ugal distortion constants has been performed with the ASYM20 program of Hedberg [23]. Tables 5 and 6 contain these rotational molecular constants for $\mathrm{HCOCN}$ and HCSCN, respectively.

\section{Results and discussion}

The equilibrium geometries of $\mathrm{HCOCN}$ and
$\mathrm{HCSCN}$ are rather similar to each other (see table 2 ), the basic difference being a considerably shorter $\mathrm{CC}$ distance in $\mathrm{HCSCN}$ compared to HCOCN. In $\mathrm{HCOCN}$ the smallest calculation (HF/4-21) predicts the $\mathrm{CC}$ distance to be about $0.02 \AA$ too short if compared to the other calculations. This is a failure of the small sp basis applied, since a larger basis with polarization functions, $6-31 G^{* *}$, produces already at the Hartree-Fock level an elongated CC bond, which changes the usual way by inclusion of electron correlation at the MP2 and MP3 levels. It is interesting to note that all ab initio calculations suggest a slightly bend $\mathrm{CCN}$ bond in both compounds, in which, as expected, the $\mathrm{CN}$ group bends away from the $\mathrm{C}=\mathrm{X}(\mathrm{X}=\mathrm{O} / \mathrm{S})$ bond. The deviation from $180^{\circ}$, corresponding to a linear chain, varies between $0.2^{\circ}$ and $2.0^{\circ}$ : for $\mathrm{HCOCN}$ the less sophisticated 4-21 sp basis gives $0.2^{\circ}$, while the HF/6$31 \mathrm{G}^{* *}$ calculation predicts $2.0^{\circ}$ (the MP results are in between). At the HF level the bending angle in $\mathrm{HCSCN}$ is smaller than in $\mathrm{HCOCN}$, but at the MP2 level they are about equal. The rotational constants calculated from these geometries are in good agreement with the measured ones (see tables 5 and 6 ), even the largest deviation is only about $1 \%$. The calculated $A$ value for HCOCN at the HF/4-21 level (where the theoretical bond lengths were systematically corrected) is considerably closer to the experimental result than that of Goddard [6] (who applied a scaling on the rotational constants themselves), while for $B$ and $C$ his estimates are slightly better. This proves that both the present correction of the geometry parameters and the correction scheme used for the rotational constants themselves by Goddard give accurate results, although probably the present approach is more preferable. (It should also be kept in mind that the calculated rotational constants refer to an equilibrium geometry, while the measured ones are subject to some vibrational effects.)

The dipole moment of HCOCN, calculated at the reference geometries, varies between 2.64 and 2.82 $D$, depending on the theoretical model applied. The calculated dipole moment of HCSCN is around 2.7 D. All these values are probably somewhat overestimated, even a $20 \%$ decrease can easily be justified [6].

As to the SQM force fields of $\mathrm{HCOCN}$ (table 3) 
Table 4

Fundamental vibrational frequencies of formyl cyanide and thioformyl cyanide (in $\mathrm{cm}^{-1}$ )

\begin{tabular}{|c|c|c|c|c|c|c|c|c|c|}
\hline \multirow[t]{4}{*}{ No. } & \multicolumn{6}{|c|}{ Formyl cyanide } & \multicolumn{3}{|c|}{$\begin{array}{l}\text { Thioformyl cyanide } \\
\text { calculated } \\
\text { HF } / 6-31 G^{* *}\end{array}$} \\
\hline & \multicolumn{4}{|c|}{ calculated $^{\text {a) }}$} & \multicolumn{2}{|l|}{ experimental } & \multirow[t]{3}{*}{ scaled } & \multirow[t]{3}{*}{ SQM } & \multirow[t]{3}{*}{ character } \\
\hline & \multirow[t]{2}{*}{$\mathrm{HF} / 4-21$} & \multicolumn{2}{|c|}{$\mathrm{HF} / 6-31 \mathrm{G}^{* *}$} & \multirow[t]{2}{*}{ character } & \multirow{2}{*}{$\begin{array}{l}\text { Clouthier } \\
\text { and Moule [2] }\end{array}$} & \multirow{2}{*}{$\begin{array}{l}\text { Judge } \\
\text { et al. [1] }\end{array}$} & & & \\
\hline & & scaled & SQM & & & & & & \\
\hline \multicolumn{10}{|c|}{$A^{\prime}($ in-plane $)$} \\
\hline 1 & 2892 & 2894 & 2996 & $\mathrm{CH}$ & - & - & 2966 & 3072 & $\mathrm{CH}$ \\
\hline 2 & 2276 & 2329 & 2222 & $\mathrm{CN}$ & 2229 & - & 2313 & 2208 & $\mathrm{CN}$ \\
\hline 3 & 1722 & 1803 & 1713 & $\mathrm{CO}$ & 1716 & - & 1336 & 1345 & $\beta \mathrm{CH}$ \\
\hline 4 & 1398 & 1363 & 1382 & $\beta \mathrm{CH}$ & - & - & 1098 & 1078 & $\mathrm{CS} ; \mathrm{CC}$ \\
\hline 5 & 922 & 896 & 914 & $\mathrm{CC} ; \beta \mathrm{CCO}$ & 914 & - & 862 & 864 & $\mathrm{CC} ; \mathrm{CS} ; \beta \mathrm{CCS}$ \\
\hline 6 & 639 & 607 & 611 & $\beta \mathrm{CCO} ; \mathrm{CC} ; \beta \mathrm{CCN}$ & - & - & 524 & 514 & $\beta \mathrm{CCS} ; \beta \mathrm{CCN}$ \\
\hline 7 & 225 & 238 & 230 & $\beta C \mathrm{CN} ; \beta \mathrm{CCO}$ & 230 & 227 & 206 & 202 & $\beta \mathrm{CCN} ; \beta \mathrm{CCS}$ \\
\hline \multicolumn{10}{|c|}{$A^{\prime \prime}$ (out-of-plane) } \\
\hline 8 & 956 & 993 & 1008 & $\gamma \mathrm{CH}$ & - & - & 843 & 853 & $\gamma \mathbf{C H}$ \\
\hline 9 & 339 & 322 & 306 & $\gamma \mathrm{CCN}$ & - & 278 & 359 & 342 & $\gamma \mathrm{CCN}$ \\
\hline
\end{tabular}

a) Results of the present study. The force fields are given in table 3 . In the approximate characterization of a normal mode, coordinates dominant according to the $M$-matrix criterion [22] are indicated by their description defined in table 1 .

Table 5

Rotational and quartic centrifugal distortion constants of formyl cyanide ${ }^{\text {a) }}$

\begin{tabular}{|c|c|c|c|c|c|c|c|c|}
\hline \multirow[t]{3}{*}{ Parameter } & \multicolumn{2}{|l|}{ Experimental } & \multicolumn{6}{|c|}{ 'l'heoretical b) } \\
\hline & \multirow[t]{2}{*}{ ref. [3] } & \multirow[t]{2}{*}{ ref. [4] } & \multirow[t]{2}{*}{$\mathrm{HF} / 4-21$} & \multicolumn{2}{|c|}{$\mathrm{HF} / 6-31 \mathrm{G}^{* *}$} & \multicolumn{2}{|l|}{ MP2 } & \multirow[t]{2}{*}{$\mathrm{MP} 3 / 6-31 \mathrm{G}^{* \mathrm{c})}$} \\
\hline & & & & direct & SQM & $6-31 \mathrm{G}^{* *}$ & $6-311 G^{* *}$ & \\
\hline$A(\mathrm{MHz})$ & 67473.540 & 67469.675 & 67308.9 & \multicolumn{2}{|c|}{68938.7} & 64433.9 & 65264.9 & 67017.7 \\
\hline$B(\mathrm{MHz})$ & 5010.190 & 5010.189 & 5070.3 & \multicolumn{2}{|c|}{5100.1} & 4950.8 & 4969.1 & 5028.5 \\
\hline$C(\mathrm{MHz})$ & 4656.598 & 4656.602 & 4715.6 & \multicolumn{2}{|c|}{4749.5} & 4597.5 & 4617.6 & 4670.1 \\
\hline$\Delta_{f}(\mathrm{kHz})$ & 2.266 & 2.272 & 2.363 & 1.843 & 2.312 & & & \\
\hline$\Delta_{J K}(\mathrm{kHz})$ & -143.104 & -143.115 & -159.31 & -108.7 & -141.3 & & & \\
\hline$\Delta_{K}(\mathbf{k H z})$ & 8990 & 7369.7 & 7628.7 & 5486.6 & 7258.9 & & & \\
\hline$\delta_{J}(\mathrm{kHz})$ & 0.3877 & 0.3897 & 0.415 & 0.299 & 0.382 & & & \\
\hline$\delta_{K}(\mathrm{kHz})$ & 34.325 & 33.194 & 26.278 & 21.37 & 27.50 & & & \\
\hline
\end{tabular}

a) Quartic centrifugal distortion constants correspond to $I^{\mathrm{r}}$ representation using $A$ reduction. For errors of the experimental values see the original publications.

b) The "direct" quartic centrifugal distortion constants were obtained by use of the unscaled, directly calculated quadratic force field. The "SQM" quartic centrifugal distortion constants were calculated using the SQM force field of table 3.

c) The MP3/6-31G" values were taken from ref. [6]; they are empirically corrected and do not refer to the directly calculated equilibrium structure.

it can be observed that the scaled force fields, calculated with the two rather different basis sets, are fairly similar. (Differences in the stretching force constants reflect mainly differences in the reference geometries of the individual force field calculations.) This means that even the compact 4-21 basis 
Table 6

Rotational and quartic centrifugal distortion constants of thioformyl cyanide *)

\begin{tabular}{|c|c|c|c|c|c|}
\hline \multirow[t]{3}{*}{ Parameter } & \multirow{3}{*}{$\begin{array}{l}\text { Experimental } \\
\text { [5] }\end{array}$} & \multicolumn{4}{|c|}{ Theoretical } \\
\hline & & \multicolumn{2}{|c|}{$\mathrm{HF} / 6-31 \mathrm{IG}^{* *}$} & \multicolumn{2}{|l|}{ MP2 } \\
\hline & & direct & SQM & $6-31 G^{* *}$ & $6-311 G^{* *}$ \\
\hline$A(\mathrm{MHz})$ & 43314.005 & \multicolumn{2}{|c|}{45049.0} & 42662.7 & 42454.2 \\
\hline$B(\mathrm{MHz})$ & 3205.677 & \multicolumn{2}{|c|}{3205.8} & 3143.0 & 3158.5 \\
\hline$C(\mathrm{MHz})$ & 2975.325 & \multicolumn{2}{|c|}{2992.8} & 2927.3 & 2939.8 \\
\hline$D_{J}(\mathrm{kHz})$ & 1.2656 & 0.977 & 1.240 & & \\
\hline$D_{J K}(\mathrm{kHz})$ & -102.02 & -82.78 & -105.8 & & \\
\hline$D_{K}(\mathrm{kHz})$ & 3756.1 & 3091.5 & 4024.0 & & \\
\hline$d_{1}(\mathrm{kHz})$ & -0.2288 & -0.165 & -0.210 & & \\
\hline$d_{2}(\mathrm{kHz})$ & -0.0083 & -0.0034 & -0.0044 & & \\
\hline
\end{tabular}

a) Quartic centrifugal distortion constants correspond to $\mathrm{I}^{\mathrm{r}}$ representation using $S$ reduction. For errors of the experimental values see the original publication. See footnote b) to table 5 .

gives accurate results. Thus in the following discussion of the force fields of $\mathrm{HCOCN}$ and $\mathrm{HCSCN}$ we can rely on previous accurate HF/4-21 SQM force field results obtained for similar compounds in judging the present results.

The SQM force fields of HCOCN and HCSCN reveal some interesting details of the electronic structure of these molecules. Both the length of the $\mathrm{CC}$ bonds $(\approx 1.47$ and $\approx 1.44 \AA$ in $\mathrm{HCOCN}$ and $\mathrm{HCSCN}$, respectively) and the diagonal CC stretching force constants $\left(\approx 4.5 \mathrm{aJ} \AA^{-2}\right.$ ) suggest a partial double bond character of CC. It is reasonable to assume that this is due to a delocalization of the electrons from the $\mathrm{CO} / \mathrm{CS}$ and $\mathrm{CN}$ bonds. The unscaled HF/4-21 CN stretching constant in HCN, calculated at $1.1515 \AA$, is $21.35 \mathrm{aJ} \AA^{-2}$ [20]. In HCOCN the $\mathrm{HF} / 4-21$ (HF/6-31 G**) results are 21.04 (24.33) aJ $\AA^{-2}$, calculated at $1.153_{4}\left(1.134_{3}\right) \AA$. In HCSCN the unscaled $\mathrm{HF} / 6-31 \mathrm{G}^{* *}$ value is $23.98 \mathrm{aJ} \AA^{-2}$ calculated at $1.136_{1} \AA$. The unscaled HF/4-21 CO force constant in formaldehyde [7] is $14.87 \mathrm{aJ} \AA^{-2}$, calculated at $1.208 \AA$, while in formyl cyanide it has a value of $15.05 \mathrm{aJ} \AA^{-2}$, calculated at $1.200 \AA$. The unscaled HF/4-21 CS stretching force constant in $\mathrm{H}_{2} \mathrm{CS}$ is 7.40 aJ $\AA^{-2}$, calculated at $1.611 \hat{A}$ [24]. The HCSCN unscaled HF/6-31G** result is 8.10 aJ $\AA^{-2}$ at $1.597 \AA$. Changes in the CN stretching constant thus can be attributed to change in the bond length, while changes of the $\mathrm{CO}$ (or $\mathrm{CS}$ ) stretching force constants are considerably smaller than it could be expected from change in the $\mathrm{CO}$ (or $\mathrm{CS}$ ) bond lengths. This suggests that the increased electron density of the $\mathrm{C}-\mathrm{C}$ bond, in both compounds, is due mainly to electrons withdrawn from the $\mathrm{CO} / \mathrm{CS}$ bonds.

The CCN in-plane bending constants of HCOCN, $\approx 0.27$, and of $\mathrm{HCSCN}, \approx 0.31 \mathrm{aJ} \mathrm{rad}^{-2}$ are about the same as the linear bending CCN force constant of benzonit rile, $0.30 \mathrm{aJ} \mathrm{rad}^{-2}$ [20]. It is also interesting to note that, at the $6-31 \mathrm{G}^{* *}$ level, the out-ofplane CCN bending force constants are larger than the in-plane ones, while the 4-2I calculation predicts the opposite for HCOCN.

The $\mathrm{CH}$ wagging force constant of the aldehyde group of acrolcin is 0.37 , and that of glyoxal is 0.35 aJ $\mathrm{rad}^{-2}$ (HF/4-21 values [7]). The values in $\mathrm{HCOCN}$ and $\mathrm{HCSCN}, \approx 0.37$ and $\approx 0.30 \mathrm{aJ} \mathrm{rad}^{-2}$, respectively, are thus perfectly regular.

Among the coupling force constants there are also some which are worth discussing. Before going into details we note that the interaction constants involving the $\mathrm{CN}$ triple bond are the only ones which might have different signs at the HF/4-21 and HF/ 6-31G** levels (see table 3 ).

The $\mathrm{CN} / \mathrm{CC}$ interaction ( $F_{4,1}$ in table 3 ) is the only significant constant in HCOCN which has substantially different values at the 4-21 and 6-31G** levels $\left(-0.18\right.$ and -0.02 aJ $\AA^{-2}$, respectively). Furthermore, this constant changes to a large positive value in $\operatorname{HCSCN}\left(0.21 \mathrm{aJ} \AA^{-2}\right)$, although most other force 
constants have very similar values in the two compounds. Thus this constant calls for a more detailed discussion. First it should be noted that the CN/CX $(\mathrm{X}=\mathrm{C}$ or $\mathrm{H}$ ) interaction constants have their own history. In $\mathrm{HCN}$ [25] and $\mathrm{CH}_{3} \mathrm{CN}$ [26], calculated at the HF/ 4-21 level, they have values of -0.3 and -0.1 aJ $\AA^{-2}$, respectively. The latter value was criticized by Duncan et al. [26], who found it experimentally to be $+0.2 \mathrm{aJ} \AA^{-2}$. A HF/4-21 SQM force field calculation of benzonitrile [20] indicated that the calculated values are principally correct. The present results suggest, however, that at the HF level there are some difficulties in calculating these force constants. This problem might, in turn, be associated with difficulties describing a triple bond adequately with a single reference wave function.

The scaled $\mathrm{HF} / 4-21 \mathrm{CO} / \mathrm{CH}$ stretching interaction force constant is 0.42 in formaldehyde [7], 0.42 in acrolein [7] and $0.33 \mathrm{aJ} \AA^{-2}$ in glyoxal [7]. In HCOCN it has a value of $0.32 \mathrm{aJ} \AA^{-2}$, thus it is most similar to glyoxal. The scaled HF/4-21 CO/CC interaction constant is 0.56 in glyoxal [7], 0.70 in acrolein [7] and the largest, $0.99 \mathrm{aJ} \AA^{-2}$ in HCOCN. (The HF/6-31G** value for HCOCN is almost exactly the same.) This is again in line with the picture showing a considerable electron flow from the $\mathrm{CO}$ to the $\mathrm{CC}$ bond in the case of HCOCN. For HCSCN the $\mathrm{CS} / \mathrm{CH}$ and $\mathrm{CS} / \mathrm{CC}$ coupling constants are 0.11 and $0.42 \mathrm{aJ} \AA^{-2}$, respectively, suggesting that a similar picture holds for this molecule, as well.

As usual, the $\mathrm{CH} / \mathrm{CC}$ interactions in $\mathrm{HCOCN}$ and HCSCN are positive (the only known exceptions involve $\mathrm{CC}$ triple bonds, as that in acetylene [27] or phenylacetylene [28]; note that these results are not considered as artifacts of the calculations). On the other hand, the scaled $\mathrm{HF} / 4-21$ value of $+0.14 \mathrm{aJ}$ $\AA^{-2}$ in HCOCN is unusually large: it is about eight times larger than the corresponding constant of glyoxal [7]. One might suggest that this is just an artifact of the HF/4-21 calculation. However, the $\mathrm{HF} / 6-31 \mathrm{G}^{\text {*** }}$ values for $\mathrm{HCOCN}$ and $\mathrm{HCSCN}$ are 0.15 and 0.11 aJ $\AA^{-2}$, respectively. Thus the large value seems to be real.

The equilibrium geometries of $\mathrm{HCOCN}$ and HCSCN are of $\mathrm{C}_{s}$ symmetry, thus their nine vibrational normal modes distribute as $7 \mathbf{a}^{\prime}+2 \mathrm{a}^{\prime \prime}$. As can be seen from table 4 , the largest deviation between the calculated and measured frequency values of
HCOCN concern $\nu_{9}$, the CCN out-of-plane bending mode. As both the calculated $\mathrm{CH}$ wagging and $\mathrm{CCN}$ out-of-plane bending diagonal force constants seem to be regular (see above), two factors could be responsible for the large discrepancy: (a) an experimental misassignment; (b) calculation of the $\gamma \mathrm{CH} /$ $\gamma \mathrm{CCN}$ interaction force constant is largely in error. Test calculations to check this second possibility show that an extreme negative interaction constant would be needed to lower the $\nu_{9}$ frequency to reach 278 $\mathrm{cm}^{-1}$. We consider this as an indication that the assignment of the out-of-plane $\mathrm{CCN}$ bending fundamental of $\mathrm{HCOCN}$ should be reconsidered. For the $\mathrm{CN}$ stretching mode $\left(\nu_{2}\right)$ the discrepancy at the HF/ 4-21 level may be due to the not perfect transferability of the respective scale factor. The calculated CC stretching frequencies of $\mathrm{HCOCN}, \nu_{5}$, are in very good agreement with the experimental value of 914 $\mathrm{cm}^{-1}$ [2]. This is somewhat surprising at the HF/ 4-21 level, since the calculated $\mathrm{CC}$ bond length is too short resulting in a too large force constant.

Precision of the present calculations is shown to be high for HCOCN (table 4). A similar precision is expected for HCSCN (the maximum error in the calculated frequencies should be about $50 \mathrm{~cm}^{-1}$ ), consequently this study should be extremely valuable in the experimental assignment of the yet unobserved vibrational spectrum of $\mathrm{HCSCN}$.

As the equilibrium geometries of HCOCN and HCSCN are planar, the calculated quartic centrifugal distortion constants depend only on the in-plane force fields. For both HCOCN and HCSCN, the poor agreement between the directly calculated HF/6$31 \mathrm{G}^{* *}$ and the measured [3-5] quartic centrifugal distortion constants improves to excellent after scaling of the force fields (see tables 5 and 6 ). Predictions based on the HF/4-21 SQM force field of HCOCN are, as expected [13], of similar accuracy. The only large discrepancy in HCOCN concerns $\delta_{K}$. From the point of view of the present calculations it should be noted that values of the $\Delta_{K}$ and $\delta_{K}$ constants, especially that of $\Delta_{K}$, are most sensitive to the diagonal $\mathrm{CCN}$ bending force constant, and are rather insensitive to the stretchings. For HCSCN the only discrepancy concerns $d_{2}$, a very small constant. A possible explanation of both discrepancies recalls that the measured centrifugal distortion constants are effective values, and that vibrations distort the cen- 
trifugal distortion constants from their equilibrium values by an unknown extent. Furthermore, such vibrational effects might affect $\delta_{K}\left(\right.$ or $d_{2}$ ) the most.

\section{Conclusions}

The present study demonstrated again the great practical value of ab initio quantum chemical calculations for the interpretation of the vibrational and rotational spectra of molecules. Necessity of scaling of the force fields calculated at different theoretical levels is well demonstrated. The quadratic scaled quantum mechanical (SQM) force fields of formyl cyanide, obtained from the direct calculated $a b$ initio Hartree-Fock SCF values by scaling them with empirical scale factors, reproduce the measured frequencies remarkably well. The calculations suggest that assignment of the out-of-plane $\mathrm{CCN}$ bending fundamental should be reconsidered. It is believed that the predicted fundamentals of HCSCN have a comparablc high accuracy, and thus they can serve a future vibrational spectroscopy study of HCSCN. The moments of inertia calculated from the equilibrium geometries and the quartic centrifugal distortion constants calculated from the SQM force fields (and the geometries) are in excellent agreement with the experimental values for both compounds.

\section{References}

[1] R.H. Judge, D.C. Moule, A. Biernacki, M. Benkel, J.M. Ross and J. Rustenburg, J. Mol. Spectry. 116 (1986) 364.

[2] D.J. Clouthier and D.C. Moule, J. Am. Chem. Soc. 109 (1987) 6259.

[3] M. Bogey, J.-L. Destombes, Y. Vallee and J.-L. Ripoll, Chem. Phys. Letters 146 (1988) 227.

[4] M. Bogey, to be published.

[5] M. Bogey, C. Demuynck, J.-L. Destombes, A. Gaumont, J.-M. Denis, Y. Vallee and J.-L. Ripoll, J. Am. Chem. Soc., in press.

[6] J.D. Goddard, Chem. Phys. Letters 132 (1986) 483.

[7] P. Pulay, G. Fogarasi, G. Pongor, J.E. Boggs and A. Vargha, J. Am. Chem. Soc. 105 (1983) 7037.

[8] G. Fogarasi and P. Pulay, Ann. Rcy. Phys. Chem. 35 (1984) 191.
[9] G. Fogarasi and P. Pulay, in: Vibrational spectra and structure, Vol. 14, ed. J.R. Durig (Elsevier, Amsterdam, 1985) pp. 125-219.

[10] G. Fogarasi and P. Pulay, J. Mol. Struct. 141 (1986) 145.

[11] P. Pulay, G. Fogarasi, F. Pang and J,E. Boggs, J. Am. Chem. Soc. 101 (1979) 2550.

[ 12] G. Wlodarczak, L. Martinache, J. Demaison and B.P. van Eijck, J. Mol. Spectry. 127 (1988) 200;

G. Wlodarczak, J. Burie, J. Demaison, K. Vormann and A.G. Császár, J. Mol. Spectry. 134 (1989) 297.

[13] A.G. Császár and G. Fogarasi, J. Chem. Phys. 89 (1988) 7646.

[14] D.A. Clabo Jr., W.D. Allen, R.B. Remington, Y. Yamaguchi and H.F. Schaefer III, Chem. Phys. 123 (1988) 187.

[15] P.C. Hariharan and J.A. Pople, J. Chem. Phys. 58 (1973) 213;

M.M. Francl, W.J. Pietro, W.J. Hehre, J.S. Binkley, M.S. Gordon, D.J. DeFrees and J.A. Pople, J. Chem. Phys. 77 (1982) 3654.

[16] E.B. Wilson Jr., J.C. Decius and P.C. Cross, Molecular vibrations (McGraw-Hill, New York, 1955);

J.K.G. Watson, in: Vibrational spectra and structure, Vol. 6, ed. J.R. Durig (Elsevier, Amsterdam, 1977) pp. 1-89.

[17] R. Krishnan, J.S. Binkley, R. Seeger and J.A. Pople, J. Chem. Phys. 72 (1980) 650;

A.D. McLean and G.S. Chandler, J. Chem. Phys. 72 (1980) 5639.

[18] M.R. Peterson and R.A. Poirier, Program MONSTERGAUSS, University of Toronto, 1981.

[19] M.J. Frisch, J.S. Binkley, H.B. Schlegel, K. Raghavachari, C.F. Melius, R.L. Martin, J.J.P. Stewart, F.W. Bobrowitz, C.M. Rohlfing, L.R. Kahn, D.J. DeFres, R. Sccger, R.A. Whiteside, D.J. Fox, E.M. Fleuder and J.A. Pople, GAUSSIAN 86, Carnegie-Mellon Quantum Chemistry Publishing Unit, Pittsburgh PA, 1984.

[20] A.G. Császár and G. Fogarasi, Spectrochim. Acta 45A (1989), in press.

[21] Y. Xie and J.E. Boggs, J. Chem. Phys. 90 (1989) 4320.

[22] P. Pulay and F. Török, Acta Chim. Hung. 44 (1965) 287; G. Keresztury and G. Jalsovszky, J. Mol. Struct. 10 (1971) 304.

[23] L. Hedberg, Abstracts of Papers, 8th Austin Symposium on Gas-Phase Molecular Structure, Austin, TX, March 1978, p. 49.

[24] A. Somogyi, G. Jalsovszky, Cs. Fülöp, J. Stark and J.E. Boggs, Spectrochim. Acta 45A (1989) 679.

[25] P. Pulay, J.-G. Lee and J.E. Boggs, J. Chem. Phys. 79 (1983) 3382.

[26] J.L. Duncan, D.C. McKean, F. Tullini, G.D. Nivellini and J.P. Pena, J. Mol. Spectry. 69 (1978) 123.

[27] P. Pulay and W. Meyer, Mol. Phys. 27 (1974) 473.

[28] A.G. Czászár, G. Fogarasi and J.E. Boggs, J. Phys. Chem., in press. 